\title{
Neural events that underlie remembering something that never happened
}

Brian Gonsalves and Ken A. Paller

Nat. Neurosci. 3, 1316-1321 (2000)

The first sentence under the heading "ERPs" on page 1317 should have read, "Subjects' responses to word-only items in the study phase were classified as 'later false memories' if subjects later incorrectly remembered having seen an item as a picture, or 'later correct rejections' if subjects correctly responded that they had not seen the picture" rather than "...'later correct rejections' if subjects correctly remembered only hearing the word." We regret the error.

\section{Long-lasting cortical plasticity in the object naming system}

Miranda van Turennout, Timothy Ellmore and Alex Martin

Nat. Neurosci. 3, 1329-1334 (2000)

The color in Fig. 3a and 4a and c reproduced poorly. The corrected figures appear below. We regret the error.

a

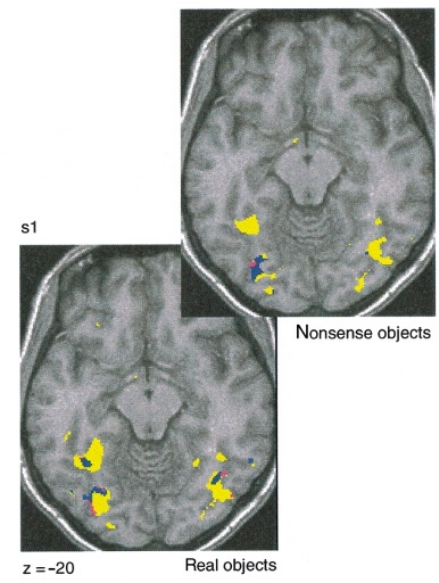

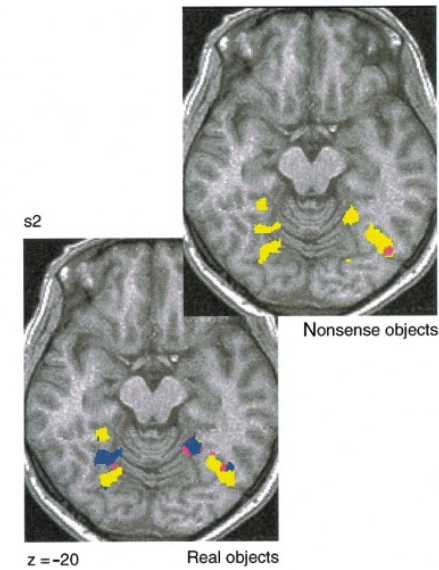

Fig. 3. Individual subject and group averaged results for nonsense objects in occipitotemporal cortex. (a) Examples of posterior regions showing repetition priming for nameable and nonsense objects in two single subjects (s1, s2). In color, regions active when viewing nonsense objects (upper slices), and viewing and silently naming real objects (lower slices) as compared to viewing visual noise stimuli. For both real and nonsense object repetition, responses were reduced at both the 30-s delay and the 3-d delay (pink). Some regions showed reduced responses at the 30-s delay only (purple).

a

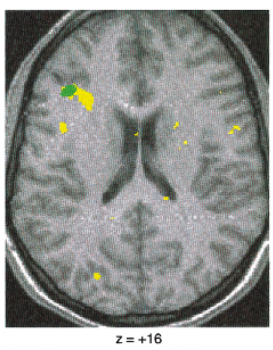

C

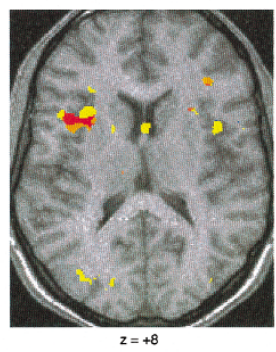

Fig. 4. Group averaged results for nameable objects in anterior regions. Increased activity for silently naming objects as compared to viewing visual noise stimuli (color) was observed in the left inferior frontal and the left insula. (a) In the left inferior frontal gyrus, activity was significantly reduced for naming repeated objects as compared to novel objects at the 3-d delay (green); reduction was not significant at the 30-s delay. (c) In the left insula, activity was significantly increased for naming repeated as compared to novel objects at both the 30-s delay (orange) and the 3-d delay (orange and red). 